\title{
Three-Dimensional Printing for Percutaneous Endoscopic Thoracic Discectomy in Thoracic Disc Herniations: Case Report and Anatomy Review
}

\author{
Zhou Chuanli ${ }^{1}$, Anthony Yeung ${ }^{2}$, Huang Hui ${ }^{1}$, Zhang Guoqing ${ }^{1}$, Gu Tong-Tong ${ }^{1}$, Ma Xuexiao ${ }^{1 *}$ and Chen Xiao-Liang ${ }^{1}$ \\ ${ }^{1}$ Department of Spine Surgery, Affiliated Hospital of Qingdao University, Qingdao 266000, P.R China \\ ${ }^{2}$ Desert Institute for Spine Care, 1635 E Myrtle Ave, Ste 400, Phoenix, AZ 85020, USA
}

"Corresponding author: Xuexiao Ma, Department of Spine Surgery, Affiliated Hospital of Qingdao University, Qingdao 266000, P.R China, Tel: (+86)18661807895; Fax: (+86)053282913018; E-mail: ma_xuexiao@126.com

Rec Date: June 19, 2017; Acc Date: July 22, 2017; Pub Date: July 26, 2017

Copyright: $\odot 2017$ Chuanli Z, et al. This is an open-access article distributed under the terms of the Creative Commons Attribution License, which permits unrestricted use, distribution, and reproduction in any medium, provided the original author and source are credited.

\begin{abstract}
Introduction: Thoracic disc herniations causing unrelenting neuralgia are relatively rare, especially cephalad to T-10 because the rib cage provides enough stability to offer relief with non-surgical methods over time. A transforaminal percutaneous endoscopic thoracic approach (PETD) however, is a feasible minimally invasive option for decompressing and removing the herniation without resorting to a more invasive and surgically more morbid open approach requiring thoracotomy and removal of the rib head through the thoracic cavity.
\end{abstract}

Method: The application of three-dimensional printing of individualized anatomic models, improves the accuracy and safety of this less invasive percutaneous operation that allows for surgical performance under local anesthesia. With the data provided by preoperative three-dimensional reconstructive CT scans, an individualized 3-D thoracic model is established by using medical mimics' software, produced with a 3-D laser printer. A more exact trajectory with a calculated skin entry point provides extra safety for a percutaneous postero-lateral approach.

A 16-year-old male, with a thoracic disc herniation and unrelenting neuralgia, is diagnosed by CT scans and magnetic resonance imaging. Non-surgical methods failed to help his severe intercostal neuralgia. Following a preoperative evaluation, a percutaneous decompression was performed, aided by 3-D printing of the thoracic spine. PETD was performed under local anesthesia.

Result: The patient had immediate resolution of his pre-operative radiating pain with no operative surgical morbidity. Extra safety is provided by operating with the patient awake.

Conclusion: Thoracic disc herniations can be decompressed safely and effectively under local anesthesia by percutaneous endoscopic thoracic decompression (PETD). $3 \mathrm{D}$ printing reconstructing the individual's anatomy at the herniation level provides an additional method for trajectory guidance. Accuracy in a trans-foraminal approach for thoracic disc herniations is critical because of the close proximity of the spinal cord and the chest cavity.

Keywords: Three-dimensional printing (3-D reconstruction); Thoracic disc herniation; Percutaneous; Endoscopic Thoracic Discectomy (PETD); Thoracic foraminal anatomy

\section{Introduction}

Thoracic spinal disorders are troublesome and present a relatively high-risk surgical condition for spine surgeons. Intercostal neuralgia caused by thoracic disc herniation that does not resolve with nonoperative treatment and require surgery comes with significant surgical complications [1].

With the development of high-resolution endoscopes, endoscopic surgical cannulas and instruments, percutaneous endoscopic thoracic discectomy (PETD) shows promise. The percutaneous transforaminal approach creates less damage to muscular and ligamentous structures, and allow for faster rehabilitation, shorter hospital stays, and earlier return to work by its minimally invasive approach, preserving bone and facet structures [2-6]. With application of three-dimensional (3-D) printing in spine surgery, individualized anatomy models improve the accuracy of the operation, especially for the percutaneous surgical approach.

We present a typical case done by PETD with the help of 3-D printing.

\section{Method}

The Ethical Review Board of Affiliate Hospital of Qingdao University approved the study. A 16-year-old boy presenting with unrelenting radiating pain from the right thorax along the ribs for 3 months was admitted. Characteristics of the pain were severe, persistent, nocturnal awakening, with unrelenting persisting radiculopathy on the right side in the T8-T9 dermatome. Physical examination indicated the patient required left recumbent positioning to obtain any relief, tenderness in the T8\T9 spinous process and the right paravertebral muscle. Hyperalgesia was present along the dermatome of the right $\mathrm{T} 8$ intercostal nerveThere was no change in 
Citation: Chuanli Z, Yeung A, Hui H, Guoqing Z, Tong-Tong G, et al. (2017) Three-Dimensional Printing for Percutaneous Endoscopic Thoracic Discectomy in Thoracic Disc Herniations: Case Report and Anatomy Review . J Spine 6: 378. doi:10.4172/2165-7939.1000378

Page 2 of 5

muscle power. Three-dimensional reconstructive CT scans and magnetic resonance imaging (MRI) confirmed a disc herniation at the T8/T9 level (Figure 1).

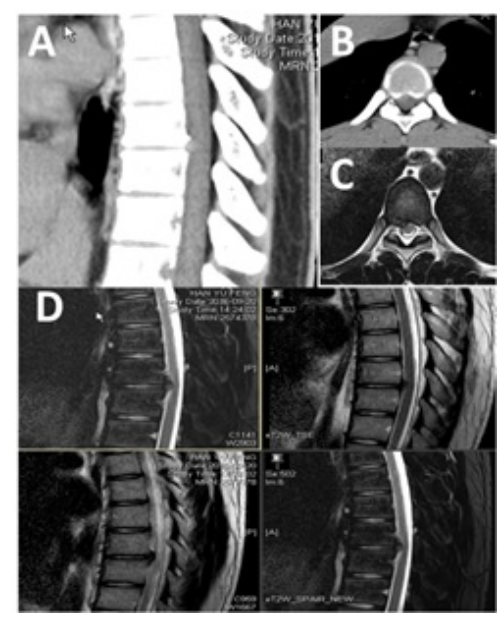

Figure 1: Sagittal (A), Transverse (B), Transverse (C) CT scan, Sagittal (D) and magnetic resonance imaging demonstrated a disc herniation at T8/T9 level and protruded into the right side of the canal.

Non-surgical treatment such as acupuncture, manipulation, massage and analgesic drugs in the physical therapy department for 3 months was not successful.

Following a preoperative evaluation, considering the young age, disc herniation, a PETD procedure was planned with the help of 3-D printing model.

\section{Design and manufacture of 3-D thoracic model}

Thoracic spine of the patient was scanned with 3-D CT scans, and images with a DICOM format were obtained (the layer and scan thickness were all $<1.25 \mathrm{~mm}$ ).
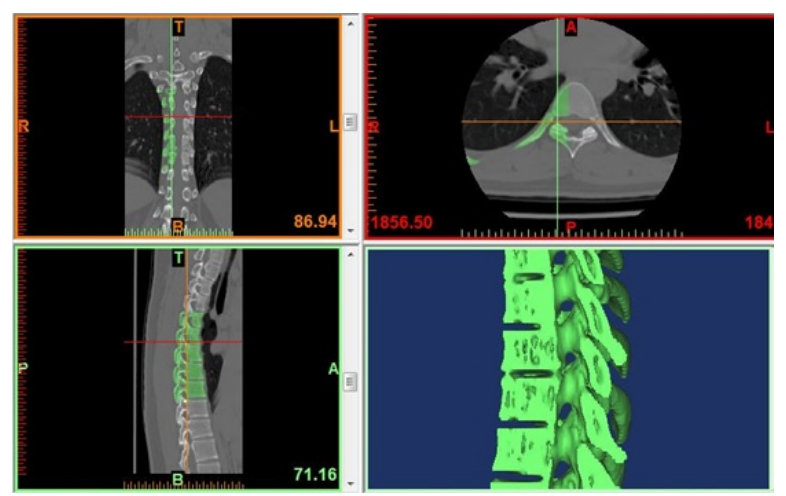

Figure 2: 3-D images of each vertebral body designed with 3-D data from mimics medical 17.0 for $\times 64$ platform.

3-D images of each vertebral body were designed with 3-D data from mimics medical 17.0 for $\times 64$ platform. PTC CREO was used to design models and Geomagic was used in reverse engineering technique. The models were saved in STL format and transmitted to a SPS600 3-D printer (provided by Qingdao University) [7,8] (Figure 2).

Pre-operative design for the puncture route for percutaneous endoscopic thoracic discectomy (PETD)

According to the measurement from CT scan and MRI in computer and 3-D printing model, we obtained an ideal entry point that identified the distance from the midline and angle incline to the head preoperatively (Figure 3).

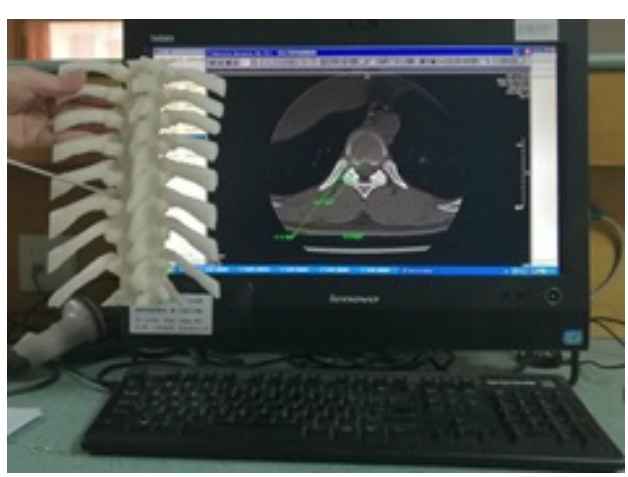

Figure 3: Ideal entry point from the CT scan and MRI and 3-D printing model preoperatively.

\section{Localization of the duty level}

Patients were positioned prone. G arm was placed on the head side and perpendicular to the patient to make sure true frontal and lateral $\mathrm{X}$ ray images. A Kirschner wire was used to localize the duty level of T8/T9 intervertebral disc space. T12 vertebral body of 3-D printing model was placed on the back to keep consistent with the real T12 column of the patient in $\mathrm{X}$ ray image, which helped to further confirm the correct duty level (Figures 4A-4C).

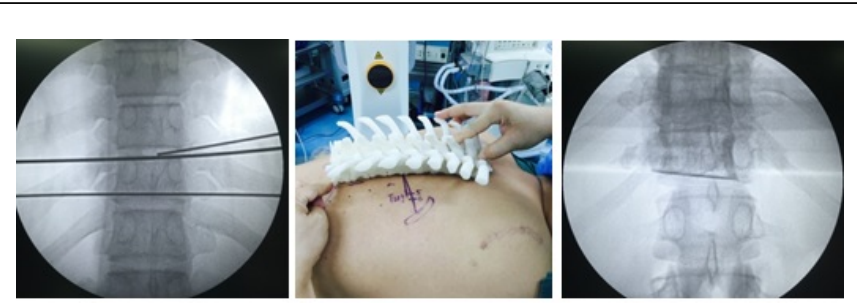

Figure 4: (A) Kirschner wire localizes the disc level in the X-ray image; (B) 3-D printing model as the localization mark; (C) T12 vertebral body of 3 -D printing model was placed on the back to keep consistent with the real T12 column of the patient in X ray image.

\section{Procedure of PETD}

An 18-gauge spinal needle with infiltrating anesthesia along the scheduled route from the skin. The injectate contained $1 \%$ lidocaine mixed with $0.5 \%$ ropivacaine. 
Citation: Chuanli Z, Yeung A, Hui H, Guoqing Z, Tong-Tong G, et al. (2017) Three-Dimensional Printing for Percutaneous Endoscopic Thoracic Discectomy in Thoracic Disc Herniations: Case Report and Anatomy Review . J Spine 6: 378. doi:10.4172/2165-7939.1000378

Page 3 of 5

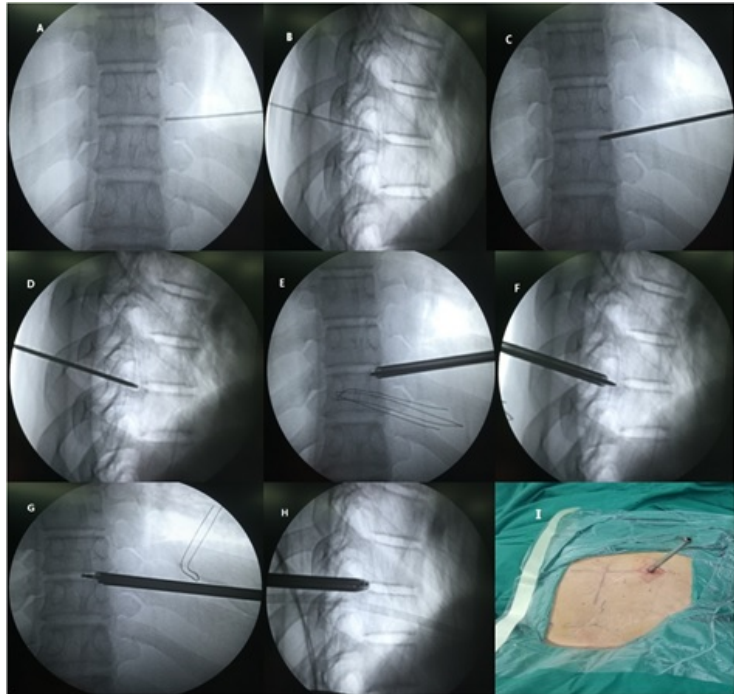

Figure 5: (A) On the antero-posterior (AP) view, the needle tip was located at the ipsilateral mid-pedicular line. (B) On the lateral view, the needle tip was at the level of the posterior disc space. $(C, D)$ Conical guiding rod was putted in along the guide wire and then serial dilators were performed to retract the muscles. (E, F) Serial reamers were applied carefully to dissect the outer and ventral part of right T8/T9 zygapophyseal joint with the eccentric reamer technique. $(G, H)$ The herniated fragment was then removed within the spinal canal with forceps slowly. (I) The working cannula was confirmed to be in a good position in the disc staying in the midpedicular line and in the disc on the lateral view ventral to the spinal canal.

Fluoroscopic verification confirmed that on the lateral view, the needle tip was at the level of the posterior disc space, and on the antero-posterior (AP) view, the needle tip was located at the ipsilateral mid-pedicular line (Figures 5A and 5B). A guide wire was inserted and docked on the foramen before the $18 \mathrm{G}$ needle was taken out, and a transverse incision with the length of $7 \mathrm{~mm}$ was made about $6 \mathrm{~cm}$ adjacent to the spinous process. A conical guiding rod was placed along the guide wire and then serial dilators were performed to dilate a path to the T 8-9-disc level. (Figures 5C and 5D). The key step of PETD was foraminoplasty with the help of serial reamers and bone drill. The serial reamers were applied carefully to dissect the outer and ventral part of right T8/T9 zygapophyseal joint with the eccentric reamer technique (Figures 5E and 5F). When foraminoplasty was accomplished, a working tube was placed in the foramen (Figure 5I). A multichannel endoscope was inserted (TESSYS ${ }^{\circledR}$ HD Foraminoscope $\mathrm{XT}$ ), under direct visualization and irrigation. The ligamentum flavum was dissected or removed to permit the endoscope to gain access to the herniation. Discectomy was then performed first by releasing the intraannular disc attachments to the sequestered disc. The herniated fragment was then removed within the spinal canal with forceps slowly (Figures $5 \mathrm{G}$ and $5 \mathrm{H}$ ).

After complete discectomy and decompression, the dura was visualized to pulse fluently. A radiofrequency bipolar coagulator was used to coagulate bleeding vessels in the annulus fibrosus. At the end, disc space, canal and intervertebral foramen would be checked again visually while gradually retrieving the working channel and endoscope (Figure 6).

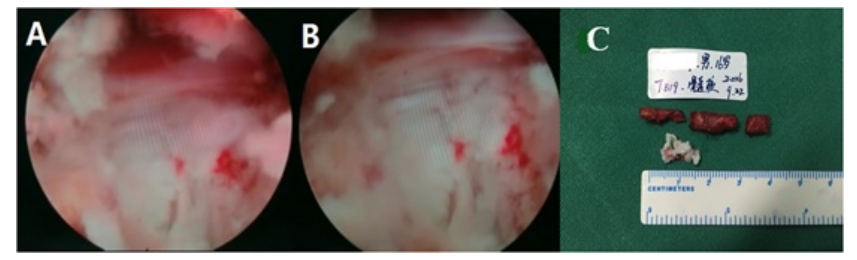

Figure 6: (A) Herniated disc was partly removed and there was a fragment at the caudal side. (B) Decompression was confirmed visually and the ventral side of dura could be seen clearly. (C) After the operation, zygapophyseal joint and rib head was confirmed partly removed, and the protruded disc was taken out. Both bone removal and disc fragment is shown in the surgical specimen.

\section{Post-operative results and imaging confirmation of the resolution of the HNP}

Radiating pain was totally gone when the patient turned over immediately after the operation, and only a bit numbness left around the zone of right $\mathrm{T} 8$ nerve root dermatome..

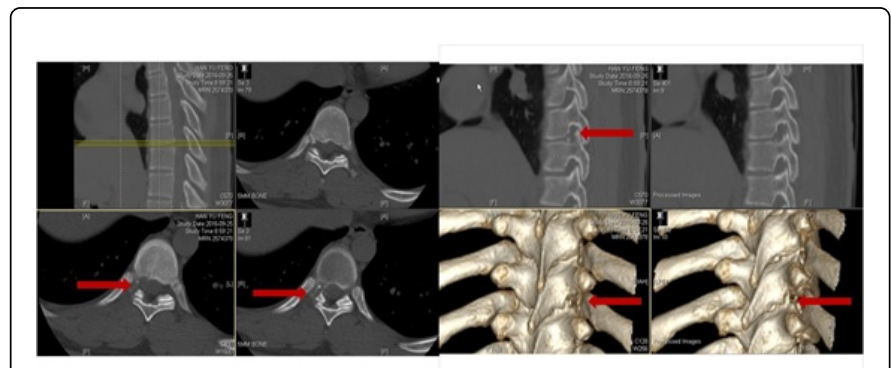

Figure 7: Post-operative three-dimensional CT scan showed the outer and ventral part of the right zygapophyseal joint decompressed.

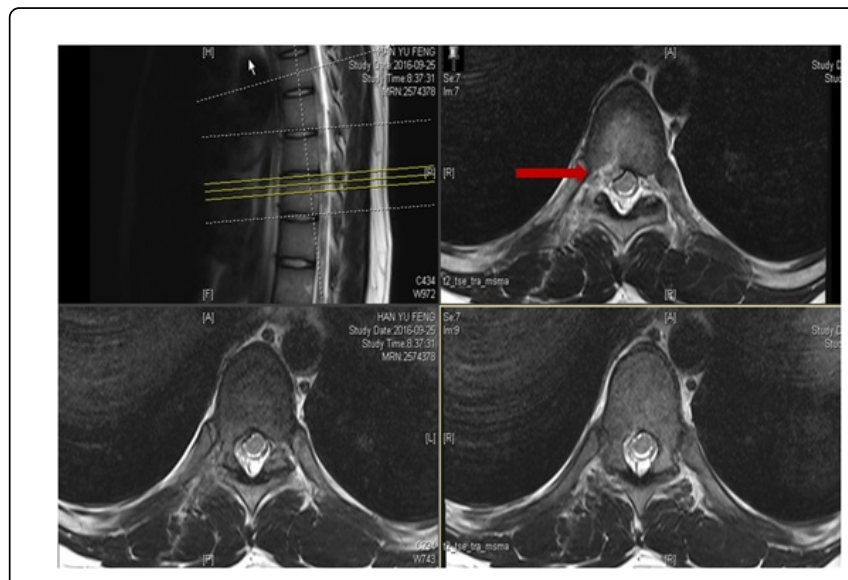

Figure 8: Post-operative MRI showed the herniated disc was taken out and only high intensity of the tissue was left. 
Citation: Chuanli Z, Yeung A, Hui H, Guoqing Z, Tong-Tong G, et al. (2017) Three-Dimensional Printing for Percutaneous Endoscopic Thoracic Discectomy in Thoracic Disc Herniations: Case Report and Anatomy Review . J Spine 6: 378. doi:10.4172/2165-7939.1000378

Page 4 of 5

Two hours later, the patient could walk by himself. No any antibiotics were used during the perioperative period. CT scans and MRI were reviewed to check the decompression. From the postoperative CT scan, the outer and ventral part of the right zygapophyseal joint were decompressed with the reamers (Figure 7) and postoperative MRI showed the herniated disc was removed, and only high intensity of the tissue was remained (Figure 8)

The cannula and endoscope must then remove the disc through the cannula. A perfect trajectory, aided by CT reconstruction of the targeted foramen is important to avoid injuring the spinal cord or entering the chest cavity.

\section{Discussion}

Herniated nucleus pulposus is less common in the thoracic region because of the immobilized structure of sternum, rib cage. When it occurs, however, conservative MDT (mechanical directional therapy) therapy would be difficult to achieve, because of the special anatomy of the thoracic canal and foramen being stabilized by the ribs.

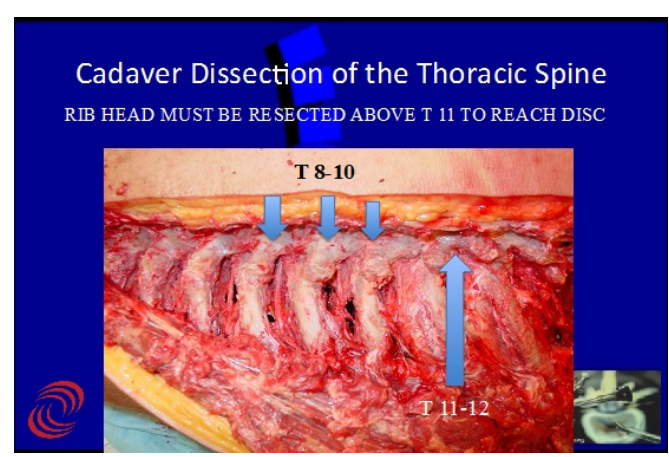

Figure 9: Cadaver anatomy of the thoracic spine showing ribs T6T12. At T8-T9 a perfect trajectory of 45 degrees with the needle and cannula at the mid pedicle of the facet in the PA view, and the serial dilating cannulas must stay at or ventral to the disc space to avoid injuring the spinal cord, but dorsal enough to avoid the chest cavity. The cannula and endoscope must then remove the disc through the cannula. A perfect trajectory, aided by CT reconstruction of the targeted foramen is important to avoid injuring the spinal cord or entering the chest cavity.

If nonoperative treatments fail, open surgery is the usual traditional neurosurgical approach. The trans-thoracic extra pleural, costotransversectomy and dorsal approaches all are associated with significant drawbacks from an exposure and technical perspective $[9,10]$. None of these approaches can be universally employed, and each is associated with approach-specific obstacles (Figure 9).

With the development of minimally invasive concept and consciousness and the advances of visualization operation system and microsurgical instruments like high-resolution endoscopes, several techniques of minimally invasive surgery were introduced.

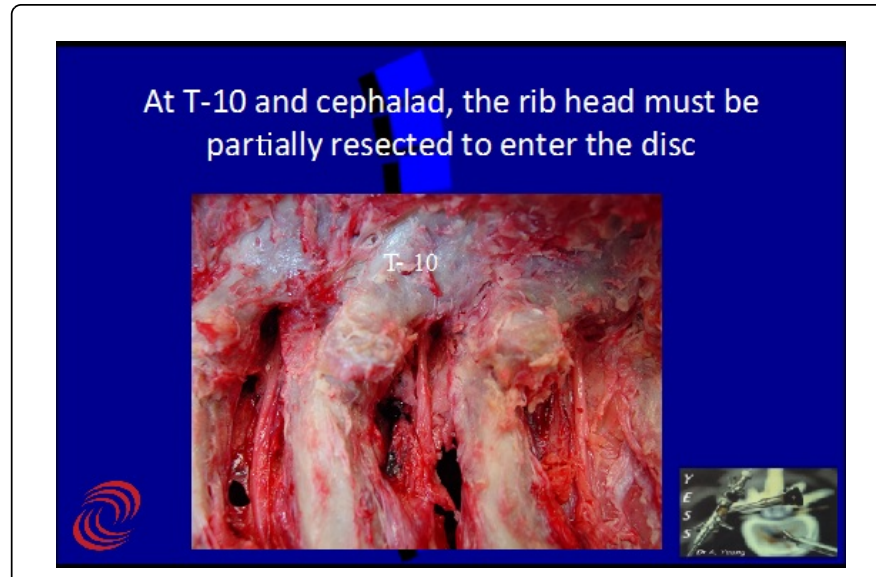

Figure 10: Cadaver dissection of the foramen T-9-T12. From T-11 to $\mathrm{L} 1$, the disc space can be accessed without removing the rib head. The foraminal space is similar to the Lumbar disc from T11-LI. The MRI will identify the level of the conus. Above T-10 careful foramenotomy is required to avoid the spinal cord and the artery of Adamkiewcz. 3-D reconstruction and Robotic guidance will aid in obtaining the perfect trajectory to the thoracic disc herniation for percutaneous discectomy.

PETD is one type of minimally invasive spinal surgery, whose procedures are derived from the percutaneous endoscopic lumbar discectomy (PELD) that would be a promising alternative to the MED technique as well as microscope-assisted technique [11-13]. Because of the differences between lumbar anatomy and thoracic anatomy, the canal and nerve root, any detailed step of PETD must be more precise from that of PELD, which has a greater degree of safety that can compensate the position of movable cannulas in the foramen. Puncture angles in three-dimensional space is also more forgiving, along with management of the zygapophyseal joint with reamer and foraminoplasty [14]. Fresh cadaver dissection to study foraminal anatomy is very helpful in understanding the anatomic variations and the best trajectory to safely enter the foramen with percutaneous endoscopic approaches (Figure 10).

From the more detailed reconstruction of individual anatomy, we exposed the posterolateral part of thoracic foramen unilaterally. A significant entity of the vertebral column was the intervertebral foramen (IVF), which had one anterosuperior part (formed by the inferior part of the pedicle and the posteroinferior part of the vertebral body) and another inferior and mobile part (formed by the posterior articular lamina covered by the yellow ligament, in the back, and by the posterolateral aspect of the intervertebral disk in front). After confirmation of the precise anatomy of the thoracic IVF as an anatomic entity, the study indicated that: Firstly, without foraminoplasty, there was not enough space to accommodate the working tube and endoscope, and accept an 18-guaze puncture needle (Figures 11A and 11B), so the outer and ventral part of the zygapophyseal joint or even dorsal and inner side of the cost transverse joint would be dissected with a reamer(Figure 11C). Secondly, the disc space was narrower and Kambin's triangle is not present as in the lumbar spine. As a result, exact angle and fenestration are critical for PETD, much more attention should be paid on the dura to prevent spinal cord injury (Figure 11D). 
Page 5 of 5

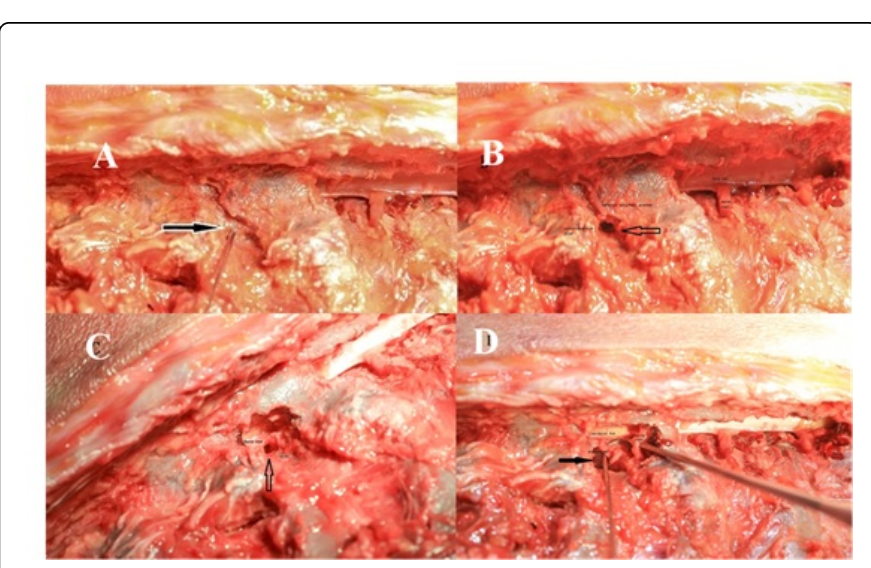

Figure 11: (A) Not enough space to accommodate the working tube and endoscope, except an 18-gauge puncture needle. (B) When the needle was taken out, only a small hole left. (C,D) Outer and ventral part of the zygapophyseal joint.

The learning curve of PETD would be steeper than that of PELD because of the above anatomy and high-risk of injury to the thoracic spinal cord. Medical Mimics software used for thoracic models could set accurate parameters and reduce data lost during process exchange between software, through which models had a higher compliance $[15,16]$. Data transformation between 3 -D CT scans and printing might have some errors of defects, which make models different from actual structures. What's more, with the help of the model, we could be familiar with each part of thoracic spine in our mind and radiation exposure by C-arm, G-arm or CT imaging navigation in traditional operations and high expense were reduced. Dr. Anthony Yeung has extensive experience in the foraminal approach for disc herniations in the lumbar spine that can carry over to the low thoracic spine from T-11 to S-1, where the foraminal anatomy is similar to lumbar anatomy. In the higher thoracic levels where herniations are more rare, the use of $3 \mathrm{D}$ reconstruction will make it easier and safer to get a perfect cannula trajectory to the thoracic herniation that makes it necessary to dilate the foramen by removing part of the rib head percutaneously from the transforaminal approach. In the future, Robotic A.I. aided by $3 \mathrm{D}$ reconstruction will ensure safer perfect trajectories demonstrated by this successful case report $[17,18]$.

\section{Conclusion}

Thoracic disc herniation can be operated safely and effectively by PETD with the help of 3-D printing. With a high accuracy in decompression and convenient operation, it provides a new method for accurate and minimally invasive operation. The future may include the use of robotics to help facilitate needle, cannula and instrument placement.

\section{Disclaimer}

There was no external funding in the preparation of this manuscript. Dr. Anthony Yeung provided his extensive experience in endoscopic trajectories and its application in the lumbar spine and comparison of the anatomy of the lumbar spine in contrast to the thoracic spine.

\section{Acknowledgement}

The authors are indebted to the imaging department of affiliated hospital of Qingdao university for data collections and analysis.

\section{References}

1. Lidar Z, Lifshutz J, Bhattacharjee S, Kurpad SN, Maiman DJ (2006) Minimally invasive, extracavitary approach for thoracic disc herniation: Technical report and preliminary results. Spine J 6: 157-163.

2. Kambin P, Brager MD (1987) Percutaneous posterolateral discectomy. Anatomy and Mechanism. Clin Orthop Relat Res 223: 145-154.

3. Kambin P, O’Brien E, Zhou L, Schaffer JL (1998) Arthroscopic micro discectomy and selective fragmentectomy. Clin Orthop Relat Res 347: 150-167.

4. Schaffer JL, Kambin P (1991) Percutaneous posterolateral lumbar discectomy and decompression with a 6.9-millimeter cannula. Analysis of operative failures and complications. J Bone Joint Surg Am 73: 822-831.

5. Hirsch JA, Singh V, Falco FJ, Benyamin RM, Manchikanti L (2009) Automated percutaneous lumbar discectomy for the contained herniated lumbar disc: A systematic assessment of evidence. Pain Physician 12: 601-620.

6. Singh V, Manchikanti L, Benyamin RM, Helm S, Hirsch JA (2009) Percutaneous lumbar laser disc decompression: A systematic review of current evidence. Pain Physician 12: 573-588.

7. Chen HL, Guo KJ, Yang HL, Wu DY, Yuan W (2016) Thoracic pedicle screw placement guide plate produce by three-dimensional (3-D) laser printing. Med Sci Monti 22: 1682-1686.

8. Gillespie EA, Matsumoto JS, Morris NE, Downey RJ, Shen RK, et al. (2016) From 3-dimentional printing to 5-dimentional printing: enhancing thoracic surgical planning and resection of complex tumors. Ann Thorac Surg 101: 1958-1962.

9. Onishi E, Yasuda T, Yamamoto H, Iwaki K, Ota S (2016) Outcomes of surgical treatment for thoracic myelopathy: A single-institutional study of 73 patients. Spine (Phila Pa 1976) 41: E1356-E1363.

10. Yang SD, Chen Q, Ning SH, Ding WY, Yang DL (2016) Modified eggshell procedure via posterior approach for sclerosing thoracic disc herniation: a preliminary study. J Orthop Surg Res 11: 102.

11. Dezawa A (2010) Development of percutaneous endoscopic approach for lumbar disc herniations. Nihon Rinsho 68: 1383-1390.

12. Wagner R, Telfeian AE, Iprenburg M, Kizok G, Gokaslan Z, et al. (2016) Tranforaminal endoscopic foraminoplasty and discectomy for the treatment of a thoracic disc herniation. World Neurosurg 90: 194-198.

13. Choi KY, Eun SS, Lee SH, Lee HY (2010) Percutaneous endoscopic thoracic discectomy: Transforaminal approach. Minim Invasive Neurosurg 53: 25-28.

14. Gkasdaris G, Tripsianis G, Kotopoulos K, Kapetanakis S (2016) Clinical anatomy and significance of the thoracic intervertebral foramen: A cadaveric study and review of the literature. J Craniovertebr Junction Spine 7: 228-235.

15. Suqimoto Y, Tanaka M, Nakahara R, Misawa H, Kunisada T, et al. (2012) Surgical treatment for congenital kyphosis correction using both spinal navigation and a 3-dimentional model. Acta Med Okayama 66: 499-502.

16. Kurenov SN, Ionita C, Sammons D, Demmy TL (2015) Threedimensional printing to facilitate anatomic study, device development, simulation, and planning in thoracic surgery. J Thorac Cardiovasc Surg 149: 973-979.

17. Yeung AT (2017) Transforaminal endoscopic decompression for painful degenerative conditions of the lumbar spine: A review of one surgeon's experience with over 10,000 cases since 1991. J Spine Neurosurg 6: 2 .

18. Yeung AT (2017) Robotics in the MIS spine surgery arena: A new role to advance the adoption of endoscopic surgery as the least invasive spine surgery procedure. J Spine 6: 374 . 